PROCEEDINGS OF THE

AMERICAN MATHEMATICAL SOCIETY

Volume 129, Number 8, Pages 2273-2275

S 0002-9939(00)05826-3

Article electronically published on November 30, 2000

\title{
AN EQUIVALENT DEFINITION OF FUNCTIONS OF THE FIRST BAIRE CLASS
}

\author{
PENG-YEE LEE, WEE-KEE TANG, AND DONGSHENG ZHAO
}

(Communicated by David Preiss)

\begin{abstract}
An equivalent definition of functions of the first Baire class in terms of $\varepsilon-\delta$ is given.
\end{abstract}

Let $X$ and $Y$ be metric spaces. A function $f: X \rightarrow Y$ is said to be of the first Baire class, or Baire-1, if $\{x: f(x) \in G\}$ is $\mathcal{F}_{\sigma}$ for every open set $G \subset Y$. The study of functions of the first Baire class dates back to 1899 in Baire's paper [1. We refer the reader to 2] and 3] for some classical results on this class of functions.

Suppose $X$ and $Y$ are complete separable metric spaces. It is well known that a function $f: X \rightarrow Y$ is Baire- 1 if and only if the restriction $f \uparrow_{P}$ of $f$ to any nonempty closed subset $P$ of $X$ has a point of continuity in $P$. (See, for instance, [2] and [3].) It is easy to see that a pointwise limit of a sequence of continuous functions from $X$ to $Y$ is Baire-1. The converse is not true. For instance, the function $\chi_{[0,1]}: \mathbb{R} \rightarrow\{0,1\}$ is Baire-1 but not a pointwise limit of a sequence of continuous functions. However, if $Y$ is a separable Banach space, then every function $f: X \rightarrow$ $Y$ that is Baire-1 is also a pointwise limit of a sequence of continuous functions.

These classifications do not involve $\varepsilon-\delta$ as in the case of the definition of continuity of a function. In this note, we give an equivalent condition for a function to be of Baire class one in terms of $\varepsilon-\delta$.

The elementary notion of continuity has numerous variants. Let $\left(X, d_{X}\right)$ and $\left(Y, d_{Y}\right)$ be complete separable metric spaces. A function $f: X \rightarrow Y$ is continuous if, for each $\varepsilon>0, x \in X$, there exist $\delta(x)>0$ such that

$$
d_{Y}(f(x), f(y))<\varepsilon
$$

whenever

$$
d_{X}(x, y)<\delta(x) \text { or } d_{X}(x, y)<\delta(y) .
$$

In this paper, we show that if the connective 'or' in $\left(^{*}\right)$ were replaced by 'and', i.e., if $(*)$ were replaced by

$$
d_{X}(x, y)<\min \{\delta(x), \delta(y)\},
$$

then we would obtain a necessary and sufficient condition for a function to be of the first Baire class.

Received by the editors June 2, 1999 and, in revised form, November 18, 1999.

2000 Mathematics Subject Classification. Primary 26A21.

Key words and phrases. Functions of the first Baire class, continuity, metric spaces.

We thank A. B. Aruffo, L. Zajiček and the referee for their comments contributing to the current proof and final statement of Theorem 1. 
Theorem 1. Suppose $f: X \rightarrow Y$ is a mapping between complete separable metric spaces $\left(X, d_{X}\right)$ and $\left(Y, d_{Y}\right)$. Then the following statements are equivalent.

(1) For any $\varepsilon>0$, there exists a positive function $\delta$ on $X$ such that

$$
d_{Y}(f(x), f(y))<\varepsilon
$$

whenever

$$
d_{X}(x, y)<\min \{\delta(x), \delta(y)\} .
$$

(2) The function $f$ is of the first Baire class.

Proof. $(1) \Rightarrow(2)$. Let $F$ be a non-empty closed subset of $X$. It suffices to show that $f \uparrow_{F}$ has a point of continuity. To this end, we prove that, for some $x \in F$,

$$
\omega_{f}(x, F)=\inf _{h>0} \sup \left\{|f(u)-f(v)|: u, v \in N_{h}(x) \cap F\right\}=0,
$$

where $N_{h}(x)=\left\{u \in X: d_{X}(u, x)<h\right\}$.

Suppose, to the contrary,

$$
\omega_{f}(x, F)>0 \text { for each } x \in F .
$$

Then we have

$$
F=\bigcup_{n=1}^{\infty} C_{n}
$$

where $C_{n}$ is the closed set $\left\{x \in F: \omega_{f}(x, F) \geq \frac{1}{n}\right\}$. By Baire's Category Theorem, there exists $n_{0} \in \mathbb{N}$ such that $C_{n_{0}}$ contains a non-empty interior $U$ in $F$. Write $I=\bar{U} \subset C_{n_{0}}$.

Now let $\delta$ be a positive function as stated in (1) that corresponds to $\varepsilon=\frac{1}{6 n_{0}}$. Put $F_{n}=\left\{x \in I: \delta(x)>\frac{1}{n}\right\}$. Then we have

$$
I=\bigcup_{n=1}^{\infty} F_{n} .
$$

Again by Baire's Category Theorem, there exists $K \in \mathbb{N}$, such that $\overline{F_{K}}$ has a nonempty interior in $I$, i.e., there exist $c \in F_{K} \cap U$ and $r>0, r<\min \left\{\frac{1}{2 K}, \delta(c)\right\}$ such that

$$
N_{r}(c) \cap I \subset \overline{F_{K}} \text {. }
$$

The proof will be complete if we have shown that

$$
\sup \left\{|f(u)-f(v)|: u, v \in N_{r}(c) \cap I\right\} \leq \frac{2}{3 n_{0}} .
$$

Indeed, if this is so, then $\omega_{f}(c, F) \leq \frac{2}{3 n_{0}}$, as $N_{r}(c) \cap I$ contains a neighbourhood of $c$ in $F$. This contradicts the fact that $c \in C_{n_{0}}$. Therefore $\omega_{f}(x, F)=0$ for some $x \in F$.

To see $(* *)$, let $y \in N_{r}(c) \cap I \subset \overline{F_{K}}$. Then there exists

$$
x \in N_{r}(c) \cap N_{\delta(y)}(y) \cap F_{K} .
$$

We note that

$$
\begin{aligned}
& d_{X}(x, y)<\min \{2 r, \delta(y)\} \leq \min \left\{\frac{1}{K}, \delta(y)\right\} \leq \min \{\delta(x), \delta(y)\}, \text { and } \\
& d_{X}(x, c)<r \leq \min \{\delta(x), \delta(c)\}
\end{aligned}
$$


Therefore, by our hypothesis,

$$
d_{Y}(f(y), f(c)) \leq d_{Y}(f(y), f(x))+d_{Y}(f(x), f(c)) \leq \frac{1}{6 n_{0}}+\frac{1}{6 n_{0}}=\frac{1}{3 n_{0}} .
$$

Consequently,

$$
\sup \left\{|f(u)-f(v)|: u, v \in N_{r}(c) \cap F \cap I\right\} \leq \frac{2}{3 n_{0}} .
$$

$(2) \Rightarrow(1)$. Suppose $f: X \rightarrow Y$ is a Baire-1 function between complete separable metric spaces $X$ and $Y$. Then $Y$ is isometric to a subset of a separable Banach space $Z$. Let $h:\left(Y, d_{Y}\right) \rightarrow\left(Z,\|\cdot\|_{Z}\right)$ be an isometry. Then $h \circ f: X \rightarrow Z$ is a Baire-1 function as well. Since $Z$ is a Banach space, $h \circ f$ is a pointwise limit of a sequence of continuous functions $g_{n}: X \rightarrow Z, n \in \mathbb{N}$.

We show that $f$ satisfies (1). Let $\varepsilon>0$ and $x \in X$ be given. For each $n \in \mathbb{N}$, there exists a function $\delta_{n}>0$ such that

$$
\left\|g_{n}(x)-g_{n}(y)\right\|_{Z}<\frac{\varepsilon}{3}
$$

whenever $d_{X}(x, y)<\delta_{n}(x)$. There also exists an integer $n_{x}$ such that

$$
\left\|g_{n}(x)-h \circ f(x)\right\|_{Z}<\frac{\varepsilon}{3}
$$

for all $n \geq n_{x}$. Define a positive function $\delta: X \rightarrow \mathbb{R}^{+}$by

$$
\delta(x)=\min _{1 \leq n \leq n_{x}} \delta_{n}(x) \text { for each } x \in X .
$$

Let $x, y \in X$ such that

$$
d_{X}(x, y)<\min \{\delta(x), \delta(y)\} .
$$

Without loss of generality, we assume that $n_{x} \leq n_{y}$; then

$$
\begin{aligned}
& \|h \circ f(x)-h \circ f(y)\|_{Z} \\
& <\left\|h \circ f(x)-g_{n_{y}}(x)\right\|_{Z}+\left\|g_{n_{y}}(x)-g_{n_{y}}(y)\right\|_{Z}+\left\|g_{n_{y}}(y)-h \circ f(y)\right\|_{Z} \\
& <\frac{\varepsilon}{3}+\left\|g_{n_{y}}(x)-g_{n_{y}}(y)\right\|_{Z}+\frac{\varepsilon}{3} .
\end{aligned}
$$

But $\left\|g_{n_{y}}(x)-g_{n_{y}}(y)\right\|_{Z}<\varepsilon / 3$ as $d_{X}(x, y) \leq \delta(y) \leq \delta_{n_{y}}(y)$. Finally, since $h$ is an isometry, $d_{Y}(f(x), f(y))=\|h \circ f(x)-h \circ f(y)\|_{Z}<\varepsilon$.

\section{REFERENCES}

[1] Baire, R., Sur les fonctions des variables réeles, Ann. Mat. Pura ed Appl. 3(1899), 1-122.

[2] Kuratowski, K., Topology, Academic Press, London, 1966. MR 36:840

[3] Natanson, I. P., Theory of functions of a real variable, Vol. 2, Frederick Ungar Publishing Co., New York, 1961. MR 26:6309

Division of Mathematics, National Institute of Education, Nanyang Technological University, 469, Bukit Timah Road, Singapore 259756

E-mail address: pylee@nie.edu.sg

Division of Mathematics, National Institute of Education, Nanyang Technological University, 469, Bukit Timah Road, Singapore 259756

E-mail address: wktang@nie.edu.sg

Division of Mathematics, National Institute of Education, Nanyang Technological University, 469, Bukit Timah Road, Singapore 259756

E-mail address: dszhao@nie.edu.sg 